\author{
Jose Tembl \\ Aida Lago \\ Teresa Sevilla \\ Pilar Solis \\ Juan Vilchez
}

\section{Migraine, patent foramen ovale and migraine triggers}

Received: 10 October 2006

Accepted in revised form: 20 November 2006

Published online: 19 February 2007
J. Tembl (西) • A. Lago • T. Sevilla

P. Solis • J. Vilchez

Neurology Service,

Universitary Hospital La Fe

Avda Campanar 21,

E-46009 Valencia, Spain

e-mail: tembl-jos@gva.es

Tel./Fax: +34961973290

\begin{abstract}
Little information exists about a causal association between PFO and migraine. Some patients identify Valsalva-provoking activities (VPA) as migraine triggers.

Therefore, we speculate about a pathogenic connection. The object of the study is to investigate the prevalence of right-to-left shunt (RLS) in a cohort of patients suffering migraine with aura (MA) and its possible association with migraine attacks triggered by VPA. We investigated the circumstances triggering the migraine attacks, in a consecutive series of 72 MA patients and in a series of migraine without aura age and gender-matched. The presence and extent of RLS was assessed by transcranial
\end{abstract}

Doppler. Massive RLS appeared in $38.9 \%$ of MA and in $6.5 \%$ of migraine without aura $(p<0.001)$. MA patients identified at least one VPA as headache trigger in $45.8 \%$. A trend was found between these triggering activities and massive RLS, both in MA group OR 2.7 [1.02-7.17] and in all migraine patients OR 2.5

[1.01-6.11]. According to our results, patients with migraine who have larger RLS tend to recognize activities that increase the extent of the shunt as a trigger of their migraine attacks.

Keywords Migraine with aura • Patent foramen ovale $\cdot$ Right-to-left shunt $\cdot$ Migraine triggers $\cdot$ Valsalva manoeuvres

\section{Introduction}

The presence of patent foramen ovale (PFO) may allow venous blood to pass from the right atrium toward arterial blood in the left atrium through the interatrial septum. Although the incidence of any degree of PFO is high among the general population, the analysis of this trait in subjects with neurological diseases has raised interest in recent years [1]. The prevalence of migraine in Western countries is rather high, with some of these patients suffering migraine with aura (MA). The focal phenomenon that appears in MA is related to a phenomenon of propa- gated cortical depression and may be triggered by various stimuli, although the pathophysiology of aura and the mechanism by which the stimuli trigger migraine attacks are still unclear [2].

One of the neurological disorders in which an elevated prevalence of PFO has been reported is MA. Some studies have described an incidence of PFO in MA which is as high as among patients with stroke of undetermined origin [3-5]. Nevertheless, it is unknown if this association is incidental or if there is some connection or relationship. The interest in clarifying these relationships is further raised, as invasive techniques such as percutaneous closure of PFO is questioned as a feasible therapy in these cases [6]. 
Theoretically, some migraine triggers such as exercise or exertion can cause Valsalva manoeuvres, thus increasing the extent of right-to-left shunt (RLS) linked to PFO. If this shunt were indeed related to the genesis of migraine attacks, patients with MA and PFO might be able to recognise these activities as triggers of their migraines. The purpose of our study was to analyse a potential relationship between these triggers, the presence of RLS and MA attacks.

\section{Patients and methods}

The study population consisted of patients diagnosed with MA selected from specific outpatient visits for headaches, the first patients being recruited in late 2002. All patients met IHS criteria for migraine with typical aura [7]. Patients with a single episode of aura were expressly excluded, even when they had a history of migraine without aura. Less common forms of migraine or probable MA (acute onset or prolonged aura) were also excluded. As part of routine clinical practice, the patients were surveyed to determine medical history and triggers of migraine attacks. In particular, triggers included those commonly accepted, such as stress, sleep, changing weather, flashes of light, specific foods and hormonal factors, as well as circumstances that induce Valsalva manoeuvres such as strenuous exercise, exertion during bowel movements, weightlifting, sudden standing, coughing or laughing crises, and sexual activity. Informed consent was obtained from the patients as required by the local ethics committee to perform a transcranial Doppler. The transcranial Doppler was used to study the vessels of Willis' polygon through the temporal window and specifically determining the presence/absence of RLS as described in the method already published and used by other authors [8]. Briefly, agitated saline achieving a microbubble emulsion was injected through an antecubital venous access. One of the middle cerebral arteries was then simultaneously monitored by transcranial Doppler and the presence of RLS was determined by the appearance of transient high-intensity signals within the first 10 cardiac cycles after injection of saline solution. These measurements were repeated at baseline and after a controlled Valsalva manoeuvre. The examination allowed confirmation of the presence of RLS, as well as semiquantitative classification of the extent of the RLS. Patients with patterns of $>25$ or uncountable signals after the Valsalva's manoeuvre ("shower" or "curtain" patterns) were defined as massive shunt [9].
Univariate analysis was used to determine the correlation between the presence of RLS and MA. In addition, univariate analysis was also employed in an attempt to identify the possible relationship between triggers (classic and those specifically investigated that produce Valsalva's manoeuvre) and the presence or absence of RLS and the degree of shunt. We established a level of alpha error at 0.05 for single comparisons and for the subgroups analysis alpha was considered both at customary 0.05 and corrected for multiple comparisons at 0.025 .

The same measurements were performed on a control series of patients with migraine without aura.

\section{Results}

We included a total of 72 patients diagnosed with MA and 31 with migraine without aura. A total of 103 patients were included with mean age $36 \pm 11.3$ years; 36 men and 67 women. Mean age was $36 \pm 12$ years (46 women, 26 men) among patients with MA and $37 \pm 9$ years (21 women, 10 men) in those with migraine without aura. No differences were found in age or distribution by sex between patients with or without aura. The most relevant data on medical history are shown in Table 1 .

In the survey on crisis triggers in patients with MA, stress was mentioned most often by 50 patients $(69.4 \%)$, followed by changes in sleep patterns $(n=37,51.4 \%)$ and changing weather conditions $(n=31,43.1 \%)$. Strenuous physical exercise was the most common situation leading to Valsalva recognised as trigger, in 18 cases $(25 \%)$. A total of 33 patients with MA (45.8\%) reported at least one of these Valsalva-provoking activities as the trigger for their crisis.

For the migraine without aura controls, the trigger reported most often was stress by 29 patients $(93.5 \%)$ followed by changing weather and variations in sleep patterns $(n=18,58.1 \%)$. Manoeuvres leading to Valsalva were reported by 12 patients $(38.7 \%)$. There were no differences with respect to MA patients (Table 2).

RLS at baseline was found in 39 patients with MA, $54.1 \%$ of those analysed. After Valsalva's manoeuvre, 44 (61.1\%) patients showed positive RLS. In comparison with patients with migraine without aura, RLS was always more

Table 1 Medical history

\begin{tabular}{lrrrrrrr}
\hline & Heart diseases & Respiratory diseases & Hypertension & Diabetes & DVT* & Smoker & Contraceptives \\
\hline MA $\dagger$ & $3(4.2 \%)$ & $2(2.8 \%)$ & $10(13.9 \%)$ & $0(0)$ & $2(2.8 \%)$ & $24(33.3 \%)$ & $15(20.8 \%)$ \\
MwA & $0(0)$ & $2(6.5 \%)$ & $4(12.9 \%)$ & $0(0)$ & $0(0)$ & $6(19.4 \%)$ & $6(19.4 \%)$ \\
\hline
\end{tabular}

*Deep vein thrombosis

$\dagger$ Migraine with aura

$\ddagger$ Migraine without aura 
Table 2 Migraine triggers

\begin{tabular}{|c|c|c|c|c|c|c|c|}
\hline & Stress & Weather & Sleep & Foods & Valsalva & Exercise & $>2 \mathrm{VPA}^{*}$ \\
\hline $\mathrm{MA} \dagger$ & $50(69.4 \%)$ & $31(43.1 \%)$ & $37(51.4 \%)$ & $19(26.4 \%)$ & $33(45.8 \%)$ & $18(25 \%)$ & $10(13.9 \%)$ \\
\hline MwA $\ddagger$ & $29(93.5 \%)$ & $18(58.1 \%)$ & $18(58.1 \%)$ & $9(29 \%)$ & $12(38.7 \%)$ & $6(19.4 \%)$ & $4(12.9 \%)$ \\
\hline
\end{tabular}

$\dagger$ Migraine with aura

\$Migraine without aura

* More than 2 Valsalva provoking activities

common in patients with MA, whether at baseline (39 vs. 6 , $p<0.001$ ) or after Valsalva's manoeuvre (44vs. $7, p<0.001)$. The semiquantitative assessment was $38.9 \%$ massive RLS in the MA group vs. $6.5 \%$ in the group without aura.

A meticulous analysis of patients who recognised situations potentially leading to a Valsalva manoeuvre as triggers indicated the following:

a) Among MA patients: 24 patients recognising these triggers showed positive RLS and 9 patients with these triggers showed negative RLS. Despite a trend, this difference was not statistically significant $(p=0.063)$.

b) Among MA patients and considering the presence of massive shunt: patients reporting these triggers were more likely to have massive RLS ( $n=17,51.5 \%)$. In contrast, 28 patients $(71 \%)$ did not recognise these triggers and did not have massive shunt $v s .11$ patients $(28.2 \%)$ without triggers and showing massive shunt (OR 2.7, 95\% CI [1.02-7.17], $p=0.043$ ).

c) When considering the entire sample, including patients with and without aura, these differences also remained. Therefore, among those reporting these triggers massive shunt was found in 18 cases (40\%) vs. 27 with lack of massive shunt $(60 \%)$. Among patients without these triggers there was a lack of massive RLS in 46 cases $(79.3 \%)$ vs. 12 cases $(12.7 \%)$ lacking these triggers and with massive RLS, for a prevalence of massive shunt of $29.1 \%$ in all 103 patients (OR 2.5, 95\% CI [1.01-6.11], $p=0.032$ ).

\section{Discussion}

The results of this study provide some support for a potential correlation between triggers, the presence of RLS and migraine attacks. According to our results, patients with migraine who have RLS tend to recognise activities that can cause Valsalva's manoeuvre, increasing the extent of shunt as a trigger of their migraine attacks.

PFO is a vestige of foetal circulation that results from a failure of the septum primum and secundum to fuse and is present in a high percentage of the unselected adult population (27\%) [10]. Since the initial comments of
Wilmshurst et al. [11, 12] on the elevated frequency of PFO among scuba divers with decompression illness and the improvement in migraine symptoms in those who had undergone closure of the foramen ovale, several series and studies on PFO and its relationship with neurological diseases have been published [1].

Perhaps the most relevant aspect has been the elevated frequency of PFO in stroke of undetermined cause [4, 13-15] and its recurrences [16, 17]. In fact, an association between stroke, PFO and migraine has been reported $[5,18$, 19]. Along with stroke, however, migraine and particularly MA have been the focus of greater interest in studies on PFO.

The arguments put forth to support a connection between the presence of PFO and the genesis of MA are essentially the frequency of the association and the effects of foramen closure on headaches. The elevated frequency of PFO among patients with MA has been reported in various studies in recent years $[4,20]$ and interatrial septal aneurysm has also been reported more often [21]. In our study, RLS was found in $61 \%$ of the MA patients and $23 \%$ of the migraine without aura patients, figures comparable to those published in the literature. It is certainly striking in our study that many patients had RLS classified as massive, i.e., with "shower" or "curtain" patterns linked to significantly larger PFO. This finding has also been reported with very similar figures in both transoesophageal echocardiography studies [22], as well as transcranial Doppler studies [3]. Nevertheless, the numeric association does not imply causality.

According to most authors, the other basic reason for defending the causal role of PFO in migraine is the improvement in migraine after foramen ovale closure. This improvement resulted in a decrease of $14 \%-80 \%$ in the frequency of migraine attacks, depending on the series, a decrease in the severity measured by disability scales and even disappearance of the migraine attacks and/or aura [23-27]. Our study cannot contribute anything of interest in this regard as it is not an interventional study.

Many authors suggest that the association between PFO and migraine is incidental and linked to hereditary factors, with no direct link. A familial aggregation of PFO among the relatives of patients with cryptogenic stroke has been reported [28]. Several studies report a tendency 
to inherit both migraines and an interatrial septum defect, and in fact the presence of septal defects appears to be transmitted in a dominant autonomic manner and significantly associated with MA [29], without this MA-PFO association being significantly linked to the female sex [30]. These findings sustain, in part, the hypothesis of a genetically determined association which would include a subgroup of MA associated to PFO, although no author has demonstrated a mechanism that could relate them.

Elegant hypotheses suggest that the presence of PFO may result in a shunt that sidesteps the pulmonary capillary filter, thus allowing some component capable of initiating the migraine attack to reach the central nervous system. Interestingly, a high prevalence of migraine has also been reported in patients with extracardiac shunts [31]. Wilmshurst and Nightingale recently provided a detailed description of this scenario [32]. The migraine triggers could be microembolisations from the venous territory [12] or, more likely, some activated molecule or particle that may elude the filtering or deactivation ability of the lung parenchyma. This role of the lungs in migraine as a filter is not new in the literature [33]. The candidate substance according to these authors would be the amines, in particular 5-hydroxytryptamine (5-HT), which is implicated in the genesis of migraine [34] and probably a platelet hyperactivation state already shown in the pathogenesis of the migraine [35]. To complete this hypothesis the capacity of the pulmonary vessels to inactivate 5-HT has also reported [36]. The series that report improvements in migraines with antiplatelet agents support this theory in part [37]. Whether or not the bypass resulting from PFO triggers the migraine when a specific threshold is exceeded or induces through chronic shunt a state of central hyperexcitability affected by other triggers is a matter of speculation. According to this scenario, patients with PFO and migraine may recognise that daily life situations that induce Valsalva manoeuvres do trigger crises by increasing the extent of RLS. This hypothesis has already been mentioned in the theoretical literature, although the question remains unanswered to our knowledge [38]. Our results would support the role of RLS as a trigger in at least some patients, due to the mechanisms mentioned. In addition, this statement would be true for both MA (where the association is particularly prevalent for reasons not yet understood) patients and for common migraine patients who have significant RLS. Nevertheless, the results only indicate a trend and the absence of a correlation would not invalidate the initial hypothesis, as it is known that patients with PFO experience shunt without pulmonary hypertension, both at rest and during activities as common as coughing [39-41]. We should again mention the high number of patients in our study who presented significant shunt at rest. In contrast to this theory, the preliminary results of the first randomised trial on PFO closure in migraine (MIST trial) did not achieve its primary endpoint for the complete elimination of headache [42].

As in similar case-control studies, a selection bias cannot be excluded and some patients with MA in particular, when triggers exist, are more likely to show up in headache clinics, although triggers were not the reason for consultation in this particular series. During data collection patients were included consecutively without considering any characteristics, except for MA according to the current diagnostic criteria. The control subjects were selected to match such characteristics as mean age and sex, and there were no significant differences in medical history between the two groups. However, in order to minimise type two error and confounding results, the control group should be increased and should be at least as large as the MA group. Another systematic error that cannot be ruled out in this design is recall bias when reporting triggers, although triggers were routinely collected in the medical interview prior to ultrasonographic study and determination of RLS, in order to minimise this bias. Finally, the type one level of error could be considered at the routine 0.05 value or could be corrected for subgroup analysis at 0.025 (Bonferroni method for multiple comparisons) in order to reduce spurious correlations. In our opinion this correction is too conservative when testing hypotheses using variables that are mutually correlated. In this case-control, exploratory study this correction (with correlated variables) might have increased the chance that true associations would not be discovered.

In conclusion, our study adds some information that supports the PFO-migraine connection, but this association remains, on the whole, unexplained. We consider it to be of maximum interest to answer a variety of questions: Is it an aetiological or accidental relation? What is the exact mechanism? and essentially, Could any new therapeutic procedure be justified?

\section{References}

1. Anzola GP (2002) Clinical impact of patent foramen ovale diagnosis with transcranial Doppler. Eur J Ultrasound $16: 11-20$
2. Lauritzen M (1994) Pathophysiology of the migraine aura. The spreading depression theory. Brain 117:199-210
3. Anzola GP, Morandi E, Casilli F, Onorato E (2006) Different degrees of right-to-left shunting predict migraine and stroke: data from 420 patients. Neurology 66:765-767 
4. Del Sette M, Angeli S, Leandri M et al (1998) Migraine with aura and right-to-left shunt on transcranial Doppler: a case-control study. Cerebrovasc Dis 8:327-330

5. Sztajzel R, Genoud D, Roth S et al (2002) Patent foramen ovale, a possible cause of symptomatic migraine: a study of 74 patients with acute ischemic stroke. Cerebrovasc Dis 13:102-106

6. Diener HC, Weimar C, Katsarava Z (2005) Patent foramen ovale: paradoxical connection to migraine and stroke. Curr Opin Neurol 18:299-304

7. International Headache Society Classification Committee (2004) The International Classification of Headache Disorders, 2nd edn. Cephalalgia 24[Suppl 1]:1-160

8. Serena J (2000) Right-to-left shunt by contrast-transcranial doppler. On web at http://perso.wanadoo.es/jserenal/Englis $\mathrm{h} \% 20$ version\%20of\%20CODICIA\%20Study/Method.htm

9. Serena J (2000) Patterns of right-toleft shunt by contrast-transcranial doppler. On web at http://perso.wanadoo.es/jserenal/Engli sh\%20version\%20of\%20CODICIA\%20Study/Patterns.htm

10. Hagen PT, Scholz DG, Edwards WD (1984) Incidence and size of patent foramen ovale during the first 10 decades of life: an autopsy study of 965 normal hearts. Mayo Clin Proc 59:17-20

11. Wilmshurst PT, Nightingale $S$, Walsh KP, Morrison WL (2000) Effect on migraine of closure of cardiac rightto-left shunts to prevent recurrence of decompression illness or stroke or for haemodynamic reasons. Lancet 356:1648-1651

12. Wilmshurst P, Nightingale $S$ (2001) Relationship between migraine and cardiac and pulmonary right-to-left shunts. Clin Sci 100:215-220

13. Bogousslavsky J, Garazi S, Jeanrenaud X et al (1996) Stroke recurrence in patients with patent foramen ovale: the Lausanne Study. Lausanne Stroke with Paradoxal Embolism Study Group. Neurology 46:1301-1305

14. Khiani R, Daly K, McCollum C (2002) Re: Clinical and imaging findings in cryptogenic stroke patients with and without patent foramen ovale. Stroke 33:2149-2150
15. Serena J, Segura T, Perez-Ayuso MJ et al (1998) The need to quantify right-toleft shunt in acute ischemic stroke: a case-control study. Stroke 29:1322-1328

16. Cujec B, Mainra R, Johnson DH (1999) Prevention of recurrent cerebral ischemic events in patients with patent foramen ovale and cryptogenic strokes or transient ischemic attacks. Can J Cardiol 15:57-64

17. Horton SC, Bunch TJ (2004) Patent foramen ovale and stroke. Mayo Clin Proc 79:79-88

18. Lamy C, Giannesini C, Zuber $\mathrm{M}$ et al (2002) Clinical and imaging findings in cryptogenic stroke patients with and without patent foramen ovale: the PFO-ASA Study. Atrial Septal Aneurysm. Stroke 33:706-711

19. Ries S, Steinke W, Neff W et al (1996) Ischemia-induced migraine from paradoxical cardioembolic stroke. Eur Neurol 36:76-78

20. Anzola GP, Magoni M, Guindani M et al (1999) Potential source of cerebral embolism in migraine with aura: a transcranial Doppler study. Neurology 52:1622-1625

21. Carerj S, Narbone MC, Zito C et al (2003) Prevalence of atrial septal aneurysm in patients with migraine: an echocardiographic study. Headache 43:725-728

22. Schwerzmann M, Nedeltchev K, Lagger F et al (2005) Prevalence and size of directly detected patent foramen ovale in migraine with aura. Neurology 65:1415-1418

23. Azarbal B, Tobis J, Suh W et al (2005) Association of interatrial shunts and migraine headaches: impact of transcatheter closure. J Am Coll Cardiol 45:489-492

24. Morandi E, Anzola GP, Angeli S et al (2003) Transcatheter closure of patent foramen ovale: a new migraine treatment? J Interv Cardiol 16:39-42

25. Post MC, Thijs V, Herroelen L, Budts WI (2004) Closure of a patent foramen ovale is associated with a decrease in prevalence of migraine. Neurology 62:1439-1440.

26. Reisman M, Christofferson RD, Jesurum J et al (2005) Migraine headache relief after transcatheter closure of patent foramen ovale. J Am Coll Cardiol 45:493-495
27. Yankovsky AE, Kuritzky A (2003) Transformation into daily migraine with aura following transcutaneous atrial septal defect closure. Headache 43:496-498

28. Arquizan C, Coste J, Touboul PJ, Mas JL (2001) Is patent foramen ovale a family trait? A transcranial Doppler sonographic study. Stroke 32:1563-1566

29. Wilmshurst PT, Pearson MJ, Nightingale $S$ et al (2004) Inheritance of persistent foramen ovale and atrial septal defects and the relation to familial migraine with aura. Heart 90:1315-1320

30. Anzola GP, Del Sette M, Rozzini L et al (2000) The migraine-PFO connection is independent of sex. Cerebrovasc Dis 10:163

31. Post MC, Letteboer TG, Mager JJ et al (2005) A pulmonary right-to-left shunt in patients with hereditary hemorrhagic telangiectasia is associated with an increased prevalence of migraine. Chest 128:2485-2489

32. Wilmshurst P, Nightingale S (2006) The role of cardiac and pulmonary pathology in migraine: a hypothesis. Headache 46:429-434

33. Sandler M (1972) Migraine: a pulmonary disease? Lancet 1:618-619

34. Bateman DN (2000) Triptans and migraine. Lancet 355:860-861

35. Kitano A, Shimomura T, Takeshima T, Takahashi K (1994) Increased 11-dehydrothromboxane B2 in migraine: platelet hyperfunction in patients with migraine during headache-free period. Headache 34:515-518

36. Gaddum JH, Hebb CO, Silver RA, Swan AA (1953) 5-Hydroxytryptamine; pharmacological action and destruction in perfused lungs. Q J Exp Physiol Cogn Med Sci 38:255-262

37. Wilmshurst PT, Nightingale $S$, Walsh KP, Morrison WL (2005) Clopidogrel reduces migraine with aura after transcatheter closure of persistent foramen ovale and atrial septal defects. Heart 91:1173-1175

38. Cheng TO (1999) Potential source of cerebral embolism in migraine with aura: a transcranial Doppler study. Neurology 53:2213-2214 
39. Dubourg O, Bourdarias JP, Farcot JC et al (1984) Contrast echocardiographic visualization of cough-induced right to left shunt through a patent foramen ovale. J Am Coll Cardiol 4:587-594

40. Langholz D, Louie EK, Konstadt SN et al (1991) Transesophageal echocardiographic demonstration of distinct mechanisms for right to left shunting across a patent foramen ovale in the absence of pulmonary hypertension. $\mathrm{J}$ Am Coll Cardiol 18:1112-1117
41. Vandenbossche JL, Englert M (1982) Effects of respiration on an atrial septal aneurysm of the fossa ovale shown by echographic study. Am Heart J 103:922-923
42. NMT Medical Inc (2005-2006) Mist Migraine Intervention with STARFlex Technology. On web at http://www.migrainemist.org/latest_news.asp 